\title{
SIMPLE LIE SUPERALGEBRAS AND NONINTEGRABLE DISTRIBUTIONS IN CHARACTERISTIC $p$
}

\author{
SOFIANE BOUARROUDJ ${ }^{1}$, DIMITRY LEITES ${ }^{2}$
}

\begin{abstract}
Recently, Grozman and Leites returned to the original Cartan's description of Lie algebras to interpret the Melikyan algebras (for $p \leq 5$ ) and several other little-known simple Lie algebras over algebraically closed fields for $p=3$ as subalgebras of Lie algebras of vector fields preserving nonintegrable distributions analogous to (or identical with) those preserved by $\mathrm{G}(2)$, $\mathrm{O}(7), \mathrm{Sp}(4)$ and $\mathrm{Sp}(10)$. The description was performed in terms of Cartan-Tanaka-Shchepochkina prolongs using Shchepochkina's algorithm and with the help of SuperLie package. Grozman and Leites also found two new series of simple Lie algebras.

Here we apply the same method to distributions preserved by one of the two exceptional simple finite dimensional Lie superalgebras over $\mathbb{C}$; for $p=3$, we obtain a series of new simple Lie superalgebras and an exceptional one.
\end{abstract}

In memory of Felix Aleksandrovich Berezin

\section{$\S 1$. INTRODUCTION}

F. A. Berezin and supersymmetries are usually associated with physics. However, Lie superalgebras - infinitesimal supersymmetries - appeared in topology at approximately the same time as the word "spin" appeared in physics and it were these examples that Berezin first had in mind. The Lie superalgebras of topologists were often the ones over fields of characteristic $p>0$, moreover, over finite fields, see [W] CL. Since the natural symmetries are usually related to simple Lie (super)algebras, and the latter are easier to study over algebraically closed fields, the attention of mathematicians became focused on these, and here we follow the trend, although it is clear that simple Lie algebras and simple Lie superalgebras (for any $p$ ) are not as interesting as their "relatives" (central extensions, algebras of derivations, etc.). Berezin was also interested in these topics but having felt that H. Galois's passionate words "je n'ai pas de temps" were applicable to him, he choose to concentrate on other things. Lately, Lie algebras and superalgebras for $p$ prime return to the scene as prime characters, see BKK, BKR.

The habitual nowadays description of simple finite dimensional Lie algebras in terms of abstract root systems, although enables one to advance rather far in the study of representations, reminds us V. Arnold's description of Leibniz's contribution to Calculus: "Leibniz's notations are so good that using them everybody can nowadays study, and even teach, Calculus without any understanding of what one is doing". In GL4, Grozman and Leites, keeping roots handy, applied Cartan's initial (now practically forgotten) description of Lie algebras, not necessarily simple ones, in terms of nonintegrable distributions these algebras preserve. They interpreted a number of ill-described simple Lie superalgebras over algebraically closed fields of characteristic $p=5$ and 3 , and discovered two (or rather three) new series of simple Lie algebras for $p=3$. These Lie algebras seemingly have no counterparts over $\mathbb{C}$ but actually they do: they preserve the same nonintegrable distributions as some of the classical simple Lie algebras (for the lack of space, this interpretation was implicit in GL4; the explicit description of the distributions in terms of Pfaff equations can be easily obtained by means of Shchepochkina's algorithm Shch] and SuperLie package [Gr]).

1991 Mathematics Subject Classification. 17B50, 70F25.

Key words and phrases. Cartan prolongation, nonholonomic manifold, Melikyan algebras, Lie superalgebras.

We are thankful to P. Grozman and I. Shchepochkina for help; DL is thankful to MPIMiS, Leipzig, for financial support and most creative environment. 
1.1. Cartan's description of Lie algebras. The method of constructing new Lie algebras used in GL4 is as follows (all nonstandard terms will be described in the main text):

(GL1) Take a simple finite dimensional Lie algebra $\mathfrak{g}$ over $\mathbb{C}$, take its form over $\mathbb{Z}$ and reduce all structure constants modulo $p$. We may assume now that $\mathfrak{g}$ is being considered over the ground field $\mathbb{K}$ of characteristic $p$.

(GL2) For each of the "simplest" $\mathbb{Z}$-gradings $\mathfrak{g}=\oplus \mathfrak{g}_{i}$ of $\mathfrak{g}$, consider a version (adjusted to the algebra of divided powers) of the Cartan-Tanaka-Shchepochkina (CTS) prolong of the "beginning" (see [Shch]) part $\mathfrak{g}=\underset{i \leq k_{0}}{\oplus} \mathfrak{g}_{i}$, where $k_{0}$ is usually equal to 0 or 1 .

(GL3) If the CTS prolong does not coincide with $\mathfrak{g}$, it contains a simple ideal. Single out this ideal.

The situation with the classification of simple finite dimensional Lie algebras and Lie superalgebras is described in the next subsection.

1.2. The Kostrikin-Shafarevich conjecture and its super counterpart. In [KS , Kostrikin and Shafarevich conjectured a description of all simple restricted finite dimensional Lie algebras $\mathfrak{g}$ over an algebraically closed field $\mathbb{K}$ of characteristic $p>7$. Dropping the restrictedness condition, a generalized KSh-conjecture states that all simple finite dimensional Lie algebras can be obtained as the end product of the following steps:

(KS1) Take a $\mathbb{Z}$-form $\mathfrak{g}_{\mathbb{Z}}$ of a simple finite dimensional Lie algebra $\mathfrak{g}$ over $\mathbb{C}$ and tensor this $\mathbb{Z}$-form by $\mathbb{K}$ over $\mathbb{Z}$ and take all simple subquotients $\mathfrak{s i}(\mathfrak{g})$ of $\mathfrak{g}_{\mathbb{Z}} \otimes_{\mathbb{Z}} \mathbb{K}$ for all such $\mathfrak{g}$;

(KS2) take an analog $\mathfrak{g}_{\mathbb{K}}$ (with an algebra of divided powers over $\mathbb{K}$ instead of polynomials) of a simple vectorial Lie algebras $\mathfrak{g}$ over $\mathbb{C}$ and take all simple subquotients $\mathfrak{s i}\left(\mathfrak{g}_{\mathbb{K}}\right)$ of $\mathfrak{g}_{\mathbb{K}}$ for all such $\mathfrak{g}$;

(KS3) take nontrivial deformations of the simple Lie algebras obtained at steps (KS1)-(KS2), if any exists.

This conjecture is now proven; it is even true for $p=7$. For $p=5$, the list of simple finite dimensional Lie algebras contains one more item: Melikyan ${ }^{1}$ algebras (and nothing else, as proven by Premet and Strade, see $[\underline{S}]$ ). For $p=3$, there is no even feeling that a complete list of simple algebras is obtained; for $p=2$, the mood is even gloomier, cf. $[\mathrm{S}$.

Super case. Even when ALS was being written (and the classification of simple vectorial Lie superalgebras was not yet even announced, see [LSh, K3]), Leites and Shchepochkina conjectured ${ }^{2}$ that the same steps (KS1)-(KS3) applied to either simple finite dimensional Lie superalgebras or the simple vectorial Lie superalgebras (everything over $\mathbb{C}$ ) yield all simple finite dimensional Lie superalgebras over an algebraically closed field $\mathbb{K}$ of characteristic $p>7$, perhaps, even for $p=7$. To prove this conjecture is a challenging problem.

1.3. Our result. Here we apply the method (GL1)-(GL3) to a simple Lie superalgebra $\mathfrak{a g}(2)$ and obtain new simple finite dimensional Lie superalgebras for $p>2$. The novelty of our examples is due to the fact that even the conjectural list of simple Lie superalgebras (sec. 1.2) only covers $p>7$. The above procedure (GL1)-(GL3) (description of deforms of the results thus obtained should follow) is our method to get new examples of simple Lie (super)algebras; to speak about completeness of the bestiarium thus obtained is too early.

We start our quest for new simple Lie superalgebras with an algebra related to $\mathfrak{g}(2)^{3}$, the latter being of interest lately for physical applications [AW].

\section{$\S 2$. BACKGROUND}

For the background on Linear Algebra in Superspaces and the list of simple Lie superalgebras, see [LSh, [K2], and also [K3] and references therein.

\footnotetext{
${ }^{1}$ For their interpretation, see [GL4.

${ }^{2}$ Delivered at various seminars and conferences starting from 1977, it was never published until now.

${ }^{3}$ We denote the exceptional Lie algebras in the same way as the serial ones, like $\mathfrak{s l}(n)$; we thus avoid confusing $\mathfrak{g}(2)$ with the second component $\mathfrak{g}_{2}$ of a $\mathbb{Z}$-graded Lie algebra $\mathfrak{g}$.
} 
2.1. Integer bases in Lie superalgebras. Let $A=\left(a_{i j}\right)$ be an $n \times n$ matrix. A Lie superalgebra $\mathfrak{g}=\mathfrak{g}(A)$ with Cartan matrix $A=\left(a_{i j}\right)$, is given by its Chevalley generators, i.e., elements $X_{i}^{ \pm}$of degree \pm 1 and $H_{i}=\left[X_{i}^{+}, X_{i}^{-}\right]$(of degree 0 ) that satisfy the relations (hereafter in similar occasions either all superscripts \pm are + or all are -$)$

$$
\left[X_{i}^{+}, X_{j}^{-}\right]=\delta_{i j} H_{i}, \quad\left[H_{i}, H_{j}\right]=0, \quad\left[H_{i}, X_{j}^{ \pm}\right]= \pm a_{i j} X_{j}^{ \pm},
$$

and additional relations $R_{i}=0$ whose left sides are implicitly described, for a general Cartan matrix, as

"the $R_{i}$ that generate the maximal ideal $I$ such that

$$
I \cap \operatorname{Span}\left(H_{i} \mid 1 \leq i \leq n\right)=0 . "
$$

For simple (finite dimensional) Lie algebras over $\mathbb{C}$, instead of implicit description (2) we have an explicit description (Serre relations) in terms of Cartan matrices. For definition of Cartan matrices of Lie superalgebras, see GL1. In particular, for all simple Lie superalgebras of the form $\mathfrak{g}=\mathfrak{g}(A)$, except for the deforms of $\mathfrak{o s p}(4 \mid 2)$, there exist bases with respect to which all structure constants are integer. In particular, this applies to $\mathfrak{a g}(2)$, first discovered by Kaplansky, see [K1C, [K2]. For presentations of $\mathfrak{a} \mathfrak{g}(2)$, see GL1.

For vectorial Lie superalgebras, integer bases are associated with $\mathbb{Z}$-forms of $\mathbb{C}[x]$ - a supercommutative superalgebra in $a$ (ordered for convenience) indeterminates $x=\left(x_{1}, \ldots, x_{a}\right)$ of which the first $m$ indeterminates are even and the rest $n$ ones are odd $(m+n=a)$. For a multi-index $\underline{r}=\left(r_{1}, \ldots, r_{a}\right)$, we set

$$
u_{i}^{r_{i}}:=\frac{x_{i}^{r_{i}}}{r_{i} !} \quad \text { and } \quad u^{\underline{r}}:=\prod_{1 \leq i \leq a} u_{i}^{r_{i}} .
$$

The idea is to formally replace fractions with $r_{i}$ ! in denominators by inseparable symbols $u_{i}^{r_{i}}$ which are well-defined over fields of prime characteristic. Clearly,

$$
u^{\underline{r}} \cdot u^{\underline{s}}=\left(\begin{array}{c}
\underline{r}+\underline{s} \\
\underline{r}
\end{array}\right) u^{\underline{r}+\underline{s}}, \quad \text { where } \quad\left(\begin{array}{c}
\underline{r}+\underline{s} \\
\underline{r}
\end{array}\right):=\prod_{1 \leq i \leq a}\left(\begin{array}{c}
r_{i}+s_{i} \\
r_{i}
\end{array}\right) .
$$

For a set of positive integers $\underline{N}=\left(N_{1}, \ldots, N_{m}\right)$, denote

$$
\begin{aligned}
& \mathcal{O}(m ; \underline{N}):=\mathbb{K}[u ; \underline{N}]:= \\
& \operatorname{Span}_{\mathbb{K}}\left(u^{\underline{r}} \mid r_{i}<p^{N_{i}} \text { for } i \leq m \text { and } r_{i}=0 \text { or } 1 \text { for } i>m\right) .
\end{aligned}
$$

As is clear from (3), $\mathbb{K}[u ; \underline{N}]$ is a subalgebra of $\mathbb{K}[u]$. The algebra $\mathbb{K}[u]$ and its subalgebras $\mathbb{K}[u ; \underline{N}]$ are called the algebras of divided powers; they are analogs of the algebra of functions.

An important feature that differs $\mathbb{K}[u]$ from $\mathbb{K}[u ; \underline{N}]$ is the number of generators: whereas $\mathbb{K}[u]$ is generated by the $u_{i}$, the algebra $\mathbb{K}[u ; \underline{N}]$ has, additionally, the generators $u_{i}^{\left(p^{k_{i}}\right)}$ for every $k_{i}$ such that $1<k_{i}<N_{i}$. Since any derivation of a given algebra is completely determined by its value on every generator of the algebra, the Lie algebra of all derivations of $\mathbb{K}[u ; \underline{N}]$ is much larger than the Lie algebra of special derivations whose generators behave like partial derivatives:

$$
\partial_{i}\left(u_{j}^{(k)}\right)=\delta_{i j} u_{j}^{(k-1)} .
$$

In what follows, we only consider special derivations, e.g., in (6).

The simple vectorial Lie algebras over $\mathbb{C}$ have only one parameter: the number of indeterminates. If Char $\mathbb{K}=p>0$, the vectorial Lie algebras acquire one more parameter: $\underline{N}$. For Lie superalgebras, $\underline{N}$ only concerns the even indeterminates. Let

$$
\mathfrak{v e c t}(m ; \underline{N} \mid n) \text { a.k.a } W(m ; \underline{N} \mid n):=\mathfrak{d e r} \mathbb{K}[u ; \underline{N}]
$$

be the general vectorial Lie algebra. 
2.2. $\mathbb{Z}$-gradings. Recall that every $\mathbb{Z}$-grading of a given vectorial algebra is determined by setting $\operatorname{deg} u_{i}=r_{i} \in \mathbb{Z}$; every $\mathbb{Z}$-grading of a given Lie superalgebra $\mathfrak{g}(A)$ is determined by setting $\operatorname{deg} X_{i}^{ \pm}=$ $\pm r_{i} \in \mathbb{Z}$.

For the Lie algebras of the form $\mathfrak{g}(A)$, we set

$$
\operatorname{deg} X_{i}^{ \pm}= \pm \delta_{i, i_{j}} \text { for any } i_{j} \text { from a selected set }\left\{i_{1}, \ldots, i_{k}\right\}
$$

and say that we have "selected" certain $k$ Chevalley generators (or respective nodes of the Dynkin graph). Yamaguchi's theorem cited below shows that, in the study of Cartan prolongs defined below, the first gradings to consider are the ones with all $r_{i}=0$ except $k$ of them $(1 \leq k \leq 2)$ "selected" Chevalley generators for which $r_{i}=1$. In this paper we consider the simplest gradings, for $k=1$.

For vectorial algebras, filtrations are more natural than gradings; the very term "vectorial" means, actually, that the algebra is endowed with a particular (Weisfeiler) filtration, see [LSh]. Unlike Lie algebras, the vectorial Lie superalgebras can sometimes be regraded into each other; various realizations as vectorial algebras are described by means of one more parameter - regrading $\underline{r}$ — with a "standard grading" as a point of reference:

$$
\begin{aligned}
& \operatorname{vect}(m ; \underline{N} \mid n ; \underline{r}) \text { a.k.a } W(m ; \underline{N} \mid n ; \underline{r}):=\mathfrak{d e r} \mathbb{K}[u ; \underline{N}], \text { where } \\
& \operatorname{deg} u_{i}=r_{i} \text { is a grading of } \mathcal{O}(m ; \underline{N} \mid n) .
\end{aligned}
$$

For $W(m ; \underline{N} \mid n)$, the standard grading is $\underline{r}=(1, \ldots, 1)$. For the contact algebras $\mathfrak{k}(2 n+1, \underline{N})$ that preserve the Pfaff equation $\alpha(X)=0$ for $X \in \mathfrak{v e c t}(2 n+1 \mid m)$, where (see [Le]

$$
\alpha= \begin{cases}d t-\sum_{i \leq n}\left(p_{i} d q_{i}-q_{i} d p_{i}\right)+\sum_{j \leq m} \theta_{j} d \theta_{j} & \text { if } p \neq 2, \\
d t+\sum_{1 \leq i \leq k} x_{i} d x_{k+i}\left\{\begin{array}{ll}
\text { for } n=2 k \text { and } x_{1}, \ldots, x_{n} \text { all even or all odd } \\
+x_{n} d x_{n} & \text { for } n=2 k+1 \text { and } x_{1}, \ldots x_{n} \text { odd }
\end{array} \quad \text { if } p=2 .\right.\end{cases}
$$

the standard grading is $\operatorname{deg} t=2$ and the degree all other indeterminates being equal to 1 .

2.3. Cartan prolongs. Let $\mathfrak{g}_{0}$ be a Lie algebra, $\mathfrak{g}_{-1}$ a $\mathfrak{g}_{0}$-module. Let us define the $\mathbb{Z}$-graded Lie algebra $\left(\mathfrak{g}_{-1}, \mathfrak{g}_{0}\right)_{*}=\oplus_{i \geq-1} \mathfrak{g}_{i}$ called the complete Cartan prolong (the result of the Cartan prolongation) of the pair $\left(\mathfrak{g}_{-1}, \mathfrak{g}_{0}\right)$. Geometrically the Cartan prolong is the maximal Lie algebra of symmetries of the $G$-structure (here: $\left.\mathfrak{g}_{0}=\operatorname{Lie}(G)\right)$ on $\mathfrak{g}_{-1}$. The components $\mathfrak{g}_{i}$ for $i>0$ are defined recursively.

First, recall that, for any (finite dimensional) vector space $V$, we have

$$
\operatorname{Hom}(V, \operatorname{Hom}(V, \ldots, \operatorname{Hom}(V, V) \ldots)) \simeq L^{i}(V, V, \ldots, V ; V),
$$

where $L^{i}$ is the space of $i$-linear maps and we have $(i+1)$-many $V$ 's on both sides. Now, we recursively define, for any $v_{1}, \ldots, v_{i+1} \in \mathfrak{g}_{-1}$ and any $i>0$ :

$$
\begin{aligned}
\mathfrak{g}_{i}= & \left\{X \in \operatorname{Hom}\left(\mathfrak{g}_{-1}, \mathfrak{g}_{i-1}\right) \mid X\left(v_{1}\right)\left(v_{2}, v_{3}, \ldots, v_{i+1}\right)=\right. \\
& \left.X\left(v_{2}\right)\left(v_{1}, v_{3}, \ldots, v_{i+1}\right)\right\} .
\end{aligned}
$$

Let the $\mathfrak{g}_{0}$-module $\mathfrak{g}_{-1}$ be faithful. Then, clearly,

$$
\left(\mathfrak{g}_{-1}, \mathfrak{g}_{0}\right)_{*}:=\oplus \mathfrak{g}_{i} \subset \mathfrak{v e c t}(m)=\mathfrak{d e r} \mathbb{K}\left[x_{1}, \ldots, x_{m}\right], \quad \text { where } m=\operatorname{dim} \mathfrak{g}_{-1} .
$$

Moreover, setting $\operatorname{deg} x_{i}=1$ for all $i$, we see that

$$
\mathfrak{g}_{i}=\left\{X \in \mathfrak{v e c t}(m) \mid \operatorname{deg} X=i,[X, \partial] \in \mathfrak{g}_{i-1} \text { for any } \partial \in \mathfrak{g}_{-1}\right\} .
$$

Now it is subject to an easy verification that the Cartan prolong $\left(\mathfrak{g}_{-1}, \mathfrak{g}_{0}\right)_{*}$ forms a subalgebra of $\mathfrak{v e c t}(m)$. (It is also easy to see that $\left(\mathfrak{g}_{-1}, \mathfrak{g}_{0}\right)_{*}$ is a Lie algebra even if $\mathfrak{g}_{-1}$ is not a faithful $\mathfrak{g}_{0}$-module; but then it can not be realized as a subalgebra of $\mathfrak{v e c t}(m)$.)

Obviously, for $p>0$, there is a series of Cartan prolongs labelled by $\underline{N}$ and the same applies to the CTS-prolongs and the partial prolongs defined in the next subsections. 
2.4. Nonholonomic manifolds. Cartan-Tanaka-Shchepochkina (CTS) prolongs. Let $M^{n}$ be an $n$-dimensional manifold. Recall that a distribution $\mathcal{D}$ on $M$ is any subbundle of the tangent bundle; $\mathcal{D}$ is said to be integrable (in a neighborhood $U$ of a point $m \in M$ ) if, for each point $x \in U$, there is a local submanifold $S$ of $U$ containing $x$ and such that the tangent bundle to $S$ is equal to $\mathcal{D}$ restricted to $S$. A criterion due to Frobenius states that $\mathcal{D}$ is integrable if and only if its sections form a Lie algebra. Let $\mathcal{D}$ be a nonintegrable distribution; then there exists a sequence of strict inclusions

$$
\mathcal{D}=\mathcal{D}_{-1} \subset \mathcal{D}_{-2} \subset \mathcal{D}_{-3} \cdots \subset \mathcal{D}_{-d}
$$

where the fiber of $\mathcal{D}_{-i}$ at a point $x \in M$ is

$$
\mathcal{D}_{-i+1}(x)+\left[\mathcal{D}_{-1}, \mathcal{D}_{-i+1}\right](x)
$$

(here $\left.\left[\mathcal{D}_{-1}, \mathcal{D}_{-i-1}\right]=\operatorname{Span}\left([X, Y] \mid X \in \Gamma\left(\mathcal{D}_{-1}\right), Y \in \Gamma\left(\mathcal{D}_{-i-1}\right)\right)\right)$ and $d$ is the least number such that

$$
\mathcal{D}_{-d}(x)+\left[\mathcal{D}_{-1}, \mathcal{D}_{-d}\right](x)=\mathcal{D}_{-d}(x) .
$$

In case $\mathcal{D}_{-d}=T M$ the distribution is called completely nonholonomic. The number $d=d(M)$ is called the nonholonomicity degree. A manifold $M$ with a distribution $\mathcal{D}$ on it will be referred to as nonholonomic one if $d(M) \neq 1$. Let

$$
n_{i}(x)=\operatorname{dim} \mathcal{D}_{-i}(x) ; \quad n_{0}(x)=0 ; \quad n_{d}(x)=n-n_{d-1} .
$$

The distribution $\mathcal{D}$ is said to be regular if all the dimensions $n_{i}$ are constants on $M$. We will only consider regular, completely nonholonomic distributions, and, moreover, satisfying certain transitivity condition (12) introduced below.

To the tangent bundle over a nonholonomic manifold $(M, \mathcal{D})$ we assign a bundle of $\mathbb{Z}$-graded nilpotent Lie algebras as follows. Fix a point $p t \in M$. The usual adic filtration by powers of the maximal ideal $\mathfrak{m}:=\mathfrak{m}_{p t}$ consisting of functions that vanish at $p t$ should be modified because distinct coordinates may have distinct "degrees". The distribution $\mathcal{D}$ induces the following filtration in $\mathfrak{m}$ :

$$
\begin{aligned}
\mathfrak{m}_{k}= & \left\{f \in \mathfrak{m} \mid X_{1}^{a_{1}} \ldots X_{n}^{a_{n}}(f)=0 \text { for any } X_{1}, \ldots, X_{n_{1}} \in \Gamma\left(\mathcal{D}_{-1}\right),\right. \\
& X_{n_{1}+1}, \ldots, X_{n_{2}} \in \Gamma\left(\mathcal{D}_{-2}\right), \ldots, X_{n_{d-1}+1}, \ldots, X_{n} \in \Gamma\left(\mathcal{D}_{-d}\right) \\
& \text { such that } \left.\sum_{1 \leq i \leq d}\left({ }_{i} \sum_{n_{i-1}<j \leq n_{i}} a_{j}\right) \leq k\right\},
\end{aligned}
$$

where $\Gamma\left(\mathcal{D}_{-j}\right)$ is the space of germs at $p t$ of sections of the bundle $\mathcal{D}_{-j}$. Now, to a filtration

$$
\mathcal{D}=\mathcal{D}_{-1} \subset \mathcal{D}_{-2} \subset \mathcal{D}_{-3} \cdots \subset \mathcal{D}_{-d}=T M,
$$

we assign the associated graded bundle

$$
\operatorname{gr}(T M)=\oplus \operatorname{gr} \mathcal{D}_{-i}, \quad \text { where } \operatorname{gr} \mathcal{D}_{-i}=\mathcal{D}_{-i} / \mathcal{D}_{-i+1}
$$

and the bracket of sections of $\operatorname{gr}(T M)$ is, by definition, the one induced by bracketing vector fields, the sections of $T M$. We assume a "transitivity condition": The Lie algebras

$$
\left.\operatorname{gr}(T M)\right|_{p t}
$$

induced at each point $p t \in M$ are isomorphic.

The grading of the coordinates (11) determines a nonstandard grading of $\mathfrak{v e c t}(n)$ (recall (10)):

$$
\begin{aligned}
& \operatorname{deg} x_{1}=\ldots=\operatorname{deg} x_{n_{1}}=1, \\
& \operatorname{deg} x_{n_{1}+1}=\ldots=\operatorname{deg} x_{n_{2}}=2, \\
& \ldots \ldots \ldots \ldots \ldots \ldots \ldots \ldots \ldots \ldots \ldots \ldots \ldots \\
& \operatorname{deg} x_{n-n_{d-1}+1}=\ldots=\operatorname{deg} x_{n}=d .
\end{aligned}
$$

Denote by $\mathfrak{v}=\underset{i \geq-d}{\oplus} \mathfrak{v}_{i}$ the algebra $\mathfrak{v e c t}(n)$ with the grading (13). One can show that the "complete prolong" of $\mathfrak{g}_{-}$to be defined shortly, i.e., $\left(\mathfrak{g}_{-}\right)_{*}:=\left(\mathfrak{g}_{-}, \tilde{\mathfrak{g}}_{0}\right)_{*} \subset \mathfrak{v}$, where $\tilde{\mathfrak{g}}_{0}:=\mathfrak{d} \mathfrak{e} \mathfrak{r}_{0} \mathfrak{g}_{-}$, preserves $\mathcal{D}$. 
For nonholonomic manifolds, an analog of the group $G$ from the term " $G$-structure", or rather of its Lie algebra, $\mathfrak{g}=\operatorname{Lie}(G)$, is the pair $\left(\mathfrak{g}_{-}, \mathfrak{g}_{0}\right)$, where $\mathfrak{g}_{0}$ is a subalgebra of the $\mathbb{Z}$-grading preserving Lie algebra of derivations of $\mathfrak{g}_{-}$, i.e., $\mathfrak{g}_{0} \subset \mathfrak{d e r}_{0} \mathfrak{g}_{-}$. If $\mathfrak{g}_{0}$ is not explicitly indicated, we assume that $\mathfrak{g}_{0}=\mathfrak{d} \mathfrak{e} \mathfrak{r}_{0} \mathfrak{g}_{-}$, i.e., is the largest possible.

Given a pair $\left(\mathfrak{g}_{-}, \mathfrak{g}_{0}\right)$ as above, define its Tanaka-Shchepochkina prolong to be the maximal subalgebra $\left(\mathfrak{g}_{-}, \mathfrak{g}_{0}\right)_{*}=\underset{k \geq-d}{\oplus} \mathfrak{g}_{k}$ of $\mathfrak{v}$ with given non-positive part $\left(\mathfrak{g}_{-}, \mathfrak{g}_{0}\right)$. For an explicit construction of the components, see Shch. If $\mathfrak{g}_{-}=\mathfrak{g}_{-1}$ the Tanaka-Shchepochkina prolong turns into its particular case, the well-known Cartan prolong.

2.5. Partial prolongs and projective structures. Let $\left(\mathfrak{g}_{-}, \mathfrak{g}_{0}\right)_{*}$ be a depth $d$ Lie algebra; $\mathfrak{h}_{1} \subset \mathfrak{g}_{1}$ be a $\mathfrak{g}_{0}$-submodule such that $\left[\mathfrak{g}_{-1}, \mathfrak{h}_{1}\right]=\mathfrak{g}_{0}$. If such $\mathfrak{h}_{1}$ exists, define the $i$ th partial prolong of $\left(\oplus_{i \leq 0} \mathfrak{g}_{i}, \mathfrak{h}_{1}\right)$ for $i \geq 2$ to be

$$
\mathfrak{h}_{i}=\left\{D \in \mathfrak{g}_{i} \mid\left[D, \mathfrak{g}_{-1}\right] \in \mathfrak{h}_{i-1}\right\} .
$$

Set $\mathfrak{h}_{i}=\mathfrak{g}_{i}$ for $i \leq 0$ and call $\mathfrak{h}_{*}=\underset{i \geq-d}{\oplus} \mathfrak{h}_{i}$ the Shchepochkina partial prolong of $\left(\underset{i \leq 0}{\oplus} \mathfrak{g}_{i}, \mathfrak{h}_{1}\right)$, see Sh14, Shch. (Of course, the partial prolong can also be defined if $\mathfrak{h}_{0}$ is contained in $\mathfrak{g}_{0}$.)

Example. The $S L(n+1)$-action on the projective space $P^{n}$ gives the embedding $\mathfrak{s l}(n+1) \subset \mathfrak{v e c t}(n)$; here $\mathfrak{s l}(n+1)$ is a partial prolong of $\mathfrak{v e c t}(n)_{i \leq 0} \oplus \mathfrak{h}_{1}$ for some $\mathfrak{h}_{1}$.

2.6. Yamaguchi's theorem. Let $\mathfrak{s}=\underset{i \geq-d}{\oplus} \mathfrak{s}_{i}$ be a simple finite dimensional Lie algebra. Let $\left(\mathfrak{s}_{-}\right)_{*}=\left(\mathfrak{s}_{-}, \mathfrak{g}_{0}\right)_{*}$ be the CTS prolong with the maximal possible $\mathfrak{g}_{0}=\mathfrak{d} \mathfrak{e} \mathfrak{r}_{0}\left(\mathfrak{s}_{-}\right)$.

Theorem $([\mathbf{Y}])$. Over $\mathbb{C}$, the isomorphism $\left(\mathfrak{s}_{-}\right)_{*} \simeq \mathfrak{s}$ holds almost always. The exceptions (cases where $\mathfrak{s}=\underset{i \geq-d}{\bigoplus} \mathfrak{s}_{i}$ is a partial prolong in $\left.\left(\mathfrak{s}_{-}\right)_{*}=\left(\mathfrak{s}_{-}, \mathfrak{g}_{0}\right)_{*}\right)$ are

1) $\mathfrak{s}$ with the grading of depth $d=1$ (in which case $\left.\left(\mathfrak{s}_{-}\right)_{*}=\mathfrak{v e c t}\left(\mathfrak{s}_{-}^{*}\right)\right)$;

2) $\mathfrak{s}$ with the grading of depth $d=2$ and $\operatorname{dim} \mathfrak{s}_{-2}=1$, i.e., with the "contact" grading, in which case $\left(\mathfrak{s}_{-}\right)_{*}=\mathfrak{k}\left(\mathfrak{s}_{-}^{*}\right)$ (these cases correspond to "selection" of the nodes on the Dynkin graph connected with the node for the maximal root on the extended graph);

$3) \mathfrak{s}$ is either $\mathfrak{s l}(n+1)$ or $\mathfrak{s p}(2 n)$ with the grading determined by "selecting" the first and the ith of simple coroots, where $1<i<n$ for $\mathfrak{s l}(n+1)$ and $i=n$ for $\mathfrak{s p}(2 n)$. (Observe that $d=2$ with $\operatorname{dim} \mathfrak{s}_{-2}>1$ for $\mathfrak{s l}(n+1)$ and $d=3$ for $\mathfrak{s p}(2 n)$.)

Moreover, the isomorphism $\left(\mathfrak{s}_{-}, \mathfrak{s}_{0}\right)_{*} \simeq \mathfrak{s}$ also holds almost always. The cases where it fails (the ones where a projective action is possible) are $\mathfrak{s l}(n+1)$ or $\mathfrak{s p}(2 n)$ with the grading determined by "selecting" only one (the first) simple coroot.

\section{§3. Our ExAmples}

Our examples, as well as Melikyan's ones, and those of [GL4], are due to the fact that, for $p$ small, the analog of Yamaguchi's theorem is false both for Lie algebras and Lie superalgebras.

Serganova and van de Leur showed ( $\underline{\mathrm{Se}}$, $\mathbf{v d L}$; $[\mathrm{Se1})$ that the Lie superalgebra $\mathfrak{g}=\mathfrak{a g}(2)$ has the following four non-equivalent Cartan matrices:
1) $\left(\begin{array}{ccc}0 & 1 & 0 \\ -1 & 2 & -3 \\ 0 & -1 & 2\end{array}\right)$
2) $\left(\begin{array}{ccc}0 & 1 & 0 \\ -1 & 0 & 3 \\ 0 & -1 & 2\end{array}\right)$
3) $\left(\begin{array}{ccc}0 & -3 & 1 \\ -3 & 0 & 2 \\ -1 & -2 & 2\end{array}\right)$
4) $\left(\begin{array}{ccc}2 & -1 & 0 \\ -3 & 0 & 2 \\ 0 & -1 & 1\end{array}\right)$

Here we only consider the simplest $\mathbb{Z}$-grading $r$ and only the first matrix.

4.1. The first Cartan matrix and $r=(1,0,0)$. Then $\mathfrak{g}=\underset{|i| \leq 2}{\oplus} \mathfrak{g}_{i}$, where $\operatorname{dim} \mathfrak{g}_{-2}=1$ and $\operatorname{sdim} \mathfrak{g}_{-1}=0 \mid 7$. Therefore, $\mathfrak{g} \subset \mathfrak{k}(1 \mid 7)$. Observe that $\mathfrak{g}_{0}=\mathfrak{g}(2) \oplus \mathfrak{z}$, where $\mathfrak{z}=\operatorname{Span}(t)$ is the center of $\mathfrak{g}_{0}$. Hereafter the elements of the Lie superalgebra of contact vector fields are given in terms of their generating functions in indeterminates introduced in (15). 
From an explicit description of $\mathfrak{g}(2)$ and its first fundamental representation in [FH] we deduce an explicit form of the non-positive elements of $\mathfrak{g}$ which we give in terms of the generating functions with respect to the contact bracket corresponding to the contact form

$$
\alpha=d t-\sum_{i=1,3,4}\left(v_{i} d w_{i}+w_{i} d v_{i}\right)+2 u d u,
$$

where the $v_{i}, w_{i}$ and $u$ are odd and notations match $\left[\mathrm{FH}\right.$, p. 354, while our $X_{i}^{+}$and $X_{i}^{-}$correspond to $X_{i}$ and $Y_{i}$ of $[\mathrm{FH}$, p. 340, respectively.

We also set $X_{3}^{ \pm}:=\left[X_{1}^{ \pm}, X_{2}^{ \pm}\right], X_{4}^{ \pm}:=\left[X_{1}^{ \pm}, X_{3}^{ \pm}\right], X_{5}^{ \pm}:=\left[X_{1}^{ \pm}, X_{4}^{ \pm}\right], X_{6}^{ \pm}:=\left[X_{2}^{ \pm}, X_{5}^{ \pm}\right]$.

To describe the $\mathfrak{g}_{0}$-module $\mathfrak{g}_{-1}=\operatorname{Span}\left(u\right.$ and $v_{i}, w_{i}$ for $\left.i=1,3,4\right)$, only the highest weight vector suffices:

\begin{tabular}{|l|l|}
\hline $\mathfrak{g}_{i}$ & the generating functions of generators, as $\mathfrak{g}_{0}$-modules \\
\hline \hline $\mathfrak{g}_{-2}$ & 1 \\
\hline $\mathfrak{g}_{-1}$ & $v_{4}$ \\
\hline $\mathfrak{g}_{0}$ & $\mathfrak{z}=\operatorname{Span}(t)$ \\
& $X_{1}^{+}=-v_{4} w_{3}-u v_{1}$ \\
& $X_{2}^{+}=v_{3} w_{1}$ \\
& $X_{1}^{-}=-v_{3} w_{4}-u w_{1}$ \\
& $X_{2}^{-}=v_{1} w_{3}$ \\
\hline
\end{tabular}

As expected, for $p=0$ and $p>3$, the CTS prolong is isomorphic to $\mathfrak{a g}(2)$.

For $p=3$, the Lie algebra $\mathfrak{g}_{0}$ is not simple, but has a simple Lie subalgebra isomorphic to $\mathfrak{p s l}(3)$ generated by $x_{1}^{ \pm}=X_{1}^{ \pm}$, and $x_{2}^{ \pm}=\left[X_{1}, X_{2}^{ \pm}\right]$and spanned by $\left\{X_{1}^{ \pm}, X_{3}^{ \pm}, X_{4}^{ \pm}, H_{1}\right\}$. Let $\widetilde{\mathfrak{g}}_{0}:=\mathfrak{p} \mathfrak{s l}(3) \oplus \mathfrak{z}$, where $\mathfrak{z}=\operatorname{Span}\left(t+v_{1} w_{1}+v_{3} w_{3}+2 v_{4} w_{4}\right)$ is the center of $\widetilde{\mathfrak{g}}_{0}$.

The $\widetilde{\mathfrak{g}}_{0}$-module $\mathfrak{g}_{1}$ splits into two irreducible components: A 0|1-dimensional, and 0|7-dimensional with lowest weight vectors, respectively:

\begin{tabular}{|l|l|}
\hline $\mathfrak{g}_{1}$ & generating function \\
\hline \hline$V_{1}^{\prime}$ & $v_{1} v_{3} w_{4}+v_{1} u w_{1}+v_{3} u w_{3}+2 v_{4} u w_{4}+v_{4} w_{1} w_{3}$ \\
$V_{1}^{\prime \prime}$ & $t w_{4}+v_{1} w_{1} w_{4}+v_{3} w_{3} w_{4}+u w_{1} w_{3}$ \\
\hline
\end{tabular}

Since $\mathfrak{g}_{1}$ generates the positive part of the CTS prolong, $\left[\mathfrak{g}_{1}, \mathfrak{g}_{-1}\right]=\widetilde{\mathfrak{g}}_{0}$, the $\widetilde{\mathfrak{g}}_{0}$-module $\mathfrak{g}_{-1}$ is irreducible, and $\left[\mathfrak{g}_{-1}, \mathfrak{g}_{-1}\right]=\mathfrak{g}_{-2}$, the standard criterion for simplicity ([]ㅛ $)$ ensures that the CTS prolong is simple. Since none of the finite dimensional simple Lie superalgebras over $\mathbb{C}$ has grading of this form, except vectorial ones, and none of vectorial simple Lie superalgebras $\mathfrak{g}$ have the simple part of $\widetilde{\mathfrak{g}}_{0}$ isomorphic to $\mathfrak{p s l}(3)$ (which only exists for $p=3$ ), we conclude that this Lie superalgebra is a new simple Lie superalgebra indigenous to $p=3$. We denote it $\mathrm{Bj}(1 ; N \mid 7)$.

The positive components of $\operatorname{Bj}(1 ; N \mid 7)$ are all of dimension 8 (the direct sums $\mathfrak{g}_{k}=\mathfrak{g}_{k}^{\prime} \oplus \mathfrak{g}_{k}^{\prime \prime}$ of irreducible $\mathfrak{g}_{0}$-modules of dimension 1 and 7 , up to parity), except the ones of the highest degree $\left(2\left(3^{N}-1\right)+3-2=2 \cdot 3^{N}-1\right)$, which are all of dimension 1 , and the second highest degree, which are all of dimension 7 . Let $V_{k}^{\prime}$ and $V_{k}^{\prime \prime}$ be the lowest weight vectors (with respect to $\mathfrak{g}_{0}$ ) of $\mathfrak{g}_{k}^{\prime}$ and 
$\mathfrak{g}_{k}^{\prime \prime}$, respectively:

\begin{tabular}{|l|l|}
\hline $\mathfrak{g}_{k}$ & generating function: $N=1$ \\
\hline \hline$V_{2}^{\prime}$ & $t^{2}+2 v_{4} u w_{1} w_{3}+v_{3} v_{4} w_{3} w_{4}+v_{1} v_{4} w_{1} w_{4}+2 v_{1} v_{3} w_{1} w_{3}+2 v_{1} v_{3} u w_{4}$ \\
$V_{2}^{\prime \prime}$ & $2 v_{1} u w_{1} w_{4}+2 v_{3} u w_{3} w_{4}+2 v_{4} w_{1} w_{3} w_{4}+t u w_{4}+2 t w_{3} w_{4}$ \\
\hline$V_{3}^{\prime}$ & $t v_{1} v_{3} w_{4}+t v_{1} u w_{1}+t v_{3} u w_{3}+2 t v_{4} u w_{4}+t v_{4} w_{1} w_{3}$ \\
$V_{3}^{\prime \prime}$ & $t^{2} w_{4}+t u w_{1} w_{3}+2 v_{1} v_{3} w_{1} w_{3} w_{4}+t v_{1} w_{3} w_{4}+t v_{3} w_{3} w_{4}+2 v_{4} u w_{3} w_{4}$ \\
\hline$V_{4}^{\prime \prime}$ & $2 v_{1} v_{3} u w_{1} w_{3} w_{4}+2 t v_{1} u w_{1} w_{4}+2 t v_{3} u w_{3} w_{4}+2 t v_{4} w_{1} w_{3} w_{4}+t^{2} u w_{4}+2 t^{2} w_{1} w_{3}$ \\
\hline$V_{5}^{\prime}$ & $2 v_{1} v_{3} v_{4} u w_{1} w_{3} w_{4}+2 t^{2} v_{1} v_{3} w_{4}+2 t^{2} v_{1} u w_{1}+2 t^{2} v_{3} u w_{3}+t^{2} v_{4} u w_{4}+2 t^{2} v_{4} w_{1} w_{3}$ \\
\hline
\end{tabular}

Similarly (the components up to 3 being the same as for $N=1$ ):

\begin{tabular}{|c|c|}
\hline $\mathfrak{g}_{k}$ & generating function: $N=2$ \\
\hline$V_{4}^{\prime}$ & $t^{3}+2 t v_{1} v_{3} u w_{4}+2 t v_{1} v_{3} w_{1} w_{3}+t v_{1} v_{4} w_{1} w_{4}+t v_{3} v_{4} w_{3} w_{4}+2 t v_{4} u w_{1} w_{3}$ \\
\hline$V_{4}^{\prime \prime}$ & $2 v_{1} v_{3} u w_{1} w_{3} w_{4}+2 t v_{1} u w_{1} w_{4}+2 t v_{3} u w_{3} w_{4}+2 t v_{4} w_{1} w_{3} w_{4}+t^{2} u w_{4}+2 t^{2} w_{1} w_{3}$ \\
\hline$V_{5}^{\prime}$ & $2 v_{1} v_{3} v_{4} u w_{1} w_{3} w_{4}+2 t^{2} v_{1} v_{3} w_{4}+2 t^{2} v_{1} u w_{1}+2 t^{2} v_{3} u w_{3}+t^{2} v_{4} u w_{4}+2 t^{2} v_{4} w_{1} w_{3}$ \\
\hline$V_{5}^{\prime \prime}$ & $t^{3} w_{4}+t^{2} v_{1} w_{1} w_{4}+t^{2} v_{3} w_{3} w_{4}+t^{2} u w_{1} w_{3}+2 t v_{1} v_{3} w_{1} w_{3} w_{4}+2 t v_{4} u w_{1} w_{3} w_{4}$ \\
\hline$V_{6}^{\prime}$ & $t^{3} u w_{4}+2 t^{3} w_{1} w_{3}+2 t^{2} v_{1} u w_{1} w_{4}+2 t^{2} v_{3} u w_{3} w_{4}+2 t^{2} v_{4} w_{1} w_{3} w_{4}+2 t v_{1} v_{3} u w_{1} w_{3} w_{4}$ \\
\hline$V_{6}^{\prime \prime}$ & $t^{4}+2 t^{2} v_{1} v_{3} u w_{4}+2 t^{2} v_{1} v_{3} w_{1} w_{3}+t^{2} v_{1} v_{4} w_{1} w_{4}+t^{2} v_{3} v_{4} w_{3} w_{4}+2 t^{2} v_{4} u w_{1} w_{3}$ \\
\hline ... & \\
\hline$V_{16}^{\prime \prime}$ & $t^{8} u w_{4}+2 t^{8} w_{1} w_{3}+2 t^{7} v_{1} u w_{1} w_{4}+2 t^{7} v_{3} u w_{3} w_{4}+2 t^{7} v_{4} w_{1} w_{3} w_{4}+2 t^{6} v_{1} v_{3} u w_{1} w_{3} w_{4}$ \\
\hline$V_{17}^{\prime}$ & $2 t^{8} v_{1} v_{3} w_{4}+2 t^{8} v_{1} u w_{1}+2 t^{8} v_{3} u w_{3}+t^{8} v_{4} u w_{4}+2 t^{8} v_{4} w_{1} w_{3}+2 t^{6} v_{1} v_{3} v_{4} u w_{1} u$ \\
\hline
\end{tabular}

Similarly (the components up to 15 being the same as for $N=2$ ):

\begin{tabular}{|c|c|}
\hline $\mathfrak{g}_{k}$ & generating function: $N=3$ \\
\hline $\begin{array}{l}V_{16}^{\prime} \\
V_{16}^{\prime \prime} \\
\end{array}$ & $\begin{array}{l}t^{8} u w_{4}+2 t^{8} w_{1} w_{3}+2 t^{7} v_{1} u w_{1} w_{4}+2 t^{7} v_{3} u w_{3} w_{4}+2 t^{7} v_{4} w_{1} w_{3} w_{4}+2 t^{6} v_{1} v_{3} u w_{1} w_{3} w_{4} \\
t^{9}+2 t^{7} v_{1} v_{3} u w_{4}+2 t^{7} v_{1} v_{3} w_{1} w_{3}+t^{7} v_{1} v_{4} w_{1} w_{4}+t^{7} v_{3} v_{4} w_{3} w_{4}+2 t^{7} v_{4} u w_{1} w_{3}\end{array}$ \\
\hline $\begin{array}{l}V_{17}^{\prime} \\
V_{17}^{\prime \prime}\end{array}$ & $\begin{array}{l}2 t^{8} v_{1} v_{3} w_{4}+2 t^{8} v_{1} u w_{1}+2 t^{8} v_{3} u w_{3}+t^{8} v_{4} u w_{4}+2 t^{8} v_{4} w_{1} w_{3}+2 t^{6} v_{1} v_{3} v_{4} u w_{1} w_{3} w_{4} \\
t^{9} w_{4}+t^{8} v_{1} w_{1} w_{4}+t^{8} v_{3} w_{3} w_{4}+t^{8} u w_{1} w_{3}+2 t^{7} v_{1} v_{3} w_{1} w_{3} w_{4}+2 t^{7} v_{4} u w_{1} w_{3} w_{4}\end{array}$ \\
\hline$\cdots$ & \\
\hline$V_{52}^{\prime \prime}$ & $t^{26} u w_{4}+2 t^{26} w_{1} w_{3}+2 t^{25} v_{1} u w_{1} w_{4}+2 t^{25} v_{3} u w_{3} w_{4}+2 t^{25} v_{4} w_{1} w_{3} w_{4}+2 t^{24} v_{1} v_{3} w_{1} w_{3} w_{4}$ \\
\hline$V_{53}^{\prime}$ & $t^{26} v_{1} v_{3} w_{4}+t^{26} v_{1} u w_{1}+t^{26} v_{3} u w_{3}+t^{26} v_{4} u w_{4}+t^{26} v_{4} w_{1} w_{3}+t^{24} v_{1} v_{3} v_{4} u w_{1} w_{3} w_{4}$ \\
\hline
\end{tabular}

Now, let us consider subalgebras of $\operatorname{Bj}(1 ; N \mid 7)$ :

(i) Let $\mathfrak{g}_{1}^{\prime}$ be generated by $V_{1}^{\prime}$, as $\widetilde{\mathfrak{g}}_{0}$-module. The component of degree 2 of the partial CTS prolong $\left(\mathfrak{g}_{-}, \mathfrak{g}_{0}, \mathfrak{g}_{1}^{\prime}\right)_{*}$ is 0 , so the corresponding Lie algebra is not simple.

(ii) Let $\mathfrak{g}_{1}^{\prime \prime}$ be generated by $V_{1}^{\prime \prime}$, as $\widetilde{\mathfrak{g}}_{0}$-module. Let $\mathrm{bj}=\left(\mathfrak{g}_{-}, \mathfrak{g}_{0}, \mathfrak{g}_{1}^{\prime \prime}\right)_{*}$ denote the partial CTS prolong, then

$$
\operatorname{dim} \mathfrak{g}_{2}=\operatorname{dim} \operatorname{Span}\left(t^{2}+2 v_{1} v_{3} u w_{3}+2 v_{1} v_{3} w_{1} w_{3}+v_{1} v_{3} w_{1} w_{4}+v_{3} v_{4} w_{3} w_{4}+2 v_{4} u w_{1} w_{3}\right)=1
$$

and $\mathfrak{g}_{3}=0$. We see that $\left[\mathfrak{g}_{1}^{\prime \prime}, \mathfrak{g}_{-1}\right]=\widetilde{\mathfrak{g}}_{0}$. The corresponding partial CTS prolong is of dimension (10|14) and the criteria for simplicity imply that bj is simple. Since none of the known Lie superalgebra has such $b_{\overline{0}} \simeq \mathfrak{s l}(2) \oplus \mathfrak{p s l}(3)$, this simple Lie superalgebra is new. Since the partial CTS prolong for $N=2$ is the same, it is an exceptional Lie superalgebra. 
Let us summarize:

4.2. Theorem. For the first Cartan matrix of $\mathfrak{a g}(2)$ and its $\mathbb{Z}$-grading $r=(100)$, the CTS prolongs return $\mathfrak{a g}(2)$, except for the case $p=3$, which yield the simple subalgebras of series $\mathrm{Bj}(1 ; N \mid 7)$, and the exceptional algebra bj.

Post scriptum. We do not regularly check arXiv news, and missed several interesting papers by Elduque cited in $\mathrm{CE}$ and almost missed (the deadline for Berezin's volume being April 15) CE (which appeared on May 15), where several new simple Lie superalgebras for $p=3$, also related to $\mathfrak{g}(2)$, are described by an approach different from ours. Elduque's superalgebras look non-isomorphic to ours.

\section{REFERENCES}

[ALS] Alekseevsky D., Leites D., Shchepochkina I., Examples of simple Lie superalgebras of vector fields, C. r. Acad. Bulg. Sci., v. 34, 1980, no. 9, 1187-1190

[AW] Atiyah, M., Witten, E., $M$-theory dynamics on a manifold of $G_{2}$ holonomy. Adv. Theor. Math. Phys. 6 (2002), no. $1,1-106$

[BKK] Belov-Kanel, A.; Kontsevich, M. Automorphisms of the Weyl algebra. Lett. Math. Phys. 74 (2005), no. 2, 181-199.

[BKR] Belov-Kanel, A.; Rowen, L. Computational aspects of polynomial identities Research Notes in Mathematics, 9. A K Peters, Ltd., Wellesley, MA, 2005. xxii+378 pp.

[BL] Bernstein J., Leites D., Invariant differential operators and irreducible representations of Lie superalgebras of vector fields. Selecta Math. Sov., v. 1, 1981, no. 2, 143-160

[C] Cartan É., Über die einfachen Transformationsgrouppen, Leipziger Berichte (1893), 395-420. Reprinted in: Euvres complètes. Partie II. (French) [Complete works. Part II] Algèbre, systèmes différentiels et problèmes d'équivalence. [Algebra, differential systems and problems of equivalence] Second edition. Éditions du Centre National de la Recherche Scientifique (CNRS), Paris, 1984.

[CL] Ciampella, A.; Lomonaco, L. A. The universal Steenrod algebra at odd primes. Comm. Algebra 32 (2004), no. 7, 2589-2607

[CE] Cunha I., Elduque A., An extended Freudenthal magic square in characteristic 3; math.RA/0605379

[FH] Fulton, W., Harris, J., Representation theory. A first course. Graduate Texts in Mathematics, 129. Readings in Mathematics. Springer-Verlag, New York, 1991. xvi+551 pp

[Gr] Grozman P., SuperLie, http://www.equaonline.com/math/SuperLie

[GL1] Grozman P., Leites D., Defining relations for classical Lie superalgebras with Cartan matrix, Czech. J. Phys., Vol. 51, 2001, no. 1, 1-22; arXiv: hep-th/9702073

[GL2] Grozman P., Leites D., SuperLie and problems (to be) solved with it. Preprint MPIM-Bonn, 2003-39 (http://www.mpim-bonn.mpg.de)

[GL4] Grozman P., Leites D., Structures of $G(2)$ type and nonintegrable distributions in characteristic $p$. Lett. Math. Phys. 74 (2005), no. 3, 229-262; arXiv: math.RT/0509400

[K1C] Kac V. G., Letter to the editors: "Classification of simple Lie superalgebras" (Funkcional. Anal. i Priložen. 9 (1975), no. 3, 91-92). (Russian; English translation: Functional Anal. Appl. 9 (1975), no. 3, $263-265$ (1976).) Funkcional. Anal. i Priložen. 10 (1976), no. 2, 93

[K2] Kac V., Lie superagebras, Adv. Math. v. 26, 1977, 8-96

[K3] Kac, Victor G. Classification of supersymmetries. Proceedings of the International Congress of Mathematicians, Vol. I (Beijing, 2002), 319-344, Higher Ed. Press, Beijing, 2002

Cheng, Shun-Jen; Kac, V., Addendum: "Generalized Spencer cohomology and filtered deformations of $\mathbb{Z}$ graded Lie superalgebras" [Adv. Theor. Math. Phys. 2 (1998), no. 5, 1141-1182; MR1688484 (2000d:17025)]. Adv. Theor. Math. Phys. 8 (2004), no. 4, 697-709.

Cantarini, N.; Cheng, S.-J.; Kac, V. Errata to: "Structure of some $\mathbb{Z}$-graded Lie superalgebras of vector fields" [Transform. Groups 4 (1999), no. 2-3, 219-272; MR1712863 (2001b:17037)] by Cheng and Kac. Transform. Groups 9 (2004), no. 4, 399-400

[KS] Kostrikin, A. I., Shafarevich, I.R., Graded Lie algebras of finite characteristic, Izv. Akad. Nauk. SSSR Ser. Mat. 33 (1969) 251-322 (in Russian); transl.: Math. USSR Izv. 3 (1969) 237-304

[Le] Lebedev A., Non-degenerate bilinear forms in characteristic 2, related contact forms, simple Lie algebras and simple superalgebras. arXiv: math.AC/0601536

[Lind] Leites D., Indecomposable representations of Lie superalgebras. In: A. N. Sissakian et. al. (ed.), Memorial volume dedicated to Misha Saveliev and Igor Luzenko, JINR, Dubna, 2000, 126-131; math.RT/0202184

[LSh] Leites D., Shchepochkina I., Classification of the simple Lie superalgebras of vector fields, preprint MPIM2003-28 (http://www.mpim-bonn.mpg.de)

[Se] Serganova, V., Automorphisms of simple Lie superalgebras. (Russian) Izv. Akad. Nauk SSSR Ser. Mat. 48 (1984), no. 3, 585-598 
[Se1] Serganova, V., On generalizations of root systems. Comm. Algebra 24 (1996), no. 13, 4281-4299

[Shch] Shchepochkina I., How to realize Lie algebras by vector fields. Theor. and Mathem. Phys. 147(3) (2006), 821-838; arXiv: math.RT/0509472

[Sh14] Shchepochkina I., Five exceptional simple Lie superalgebras of vector fields and their fourteen regradings. Representation Theory (electronic journal of AMS), v. 3, 1999, 3 (1999), 373-415; arXiv: hep-th/9702121

[S] Strade, H. Simple Lie algebras over fields of positive characteristic. I. Structure theory. de Gruyter Expositions in Mathematics, 38. Walter de Gruyter \& Co., Berlin, 2004. viii+540 pp.

[vdL] Leur Johan van de., Contragredient Lie superalgebras of finite growth (Ph.D. thesis) Utrecht, 1986; a short version published in Commun. in Alg., v. 17, 1989, 1815-1841

[W] Wood, R. M. W. Problems in the Steenrod algebra. Bull. London Math. Soc. 30 (1998), no. 5, 449-517

[Y] Yamaguchi K., Differential systems associated with simple graded Lie algebras. Progress in differential geometry, Adv. Stud. Pure Math., 22, Math. Soc. Japan, Tokyo, 1993, 413-494

${ }^{1}$ Department of Mathematics, United Arab Emirates University, Al Ain, Po. Box: 17551; Bouar-

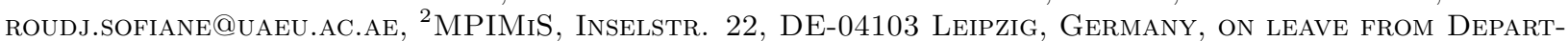
ment of Mathematics, University of Stockholm, Roslagsv. 101, Kräftriket hus 6, SE-104 05 StockHOLM, SWeden; MLEITES@MATH.Su.SE, Leites@Mis.MPG.DE 